
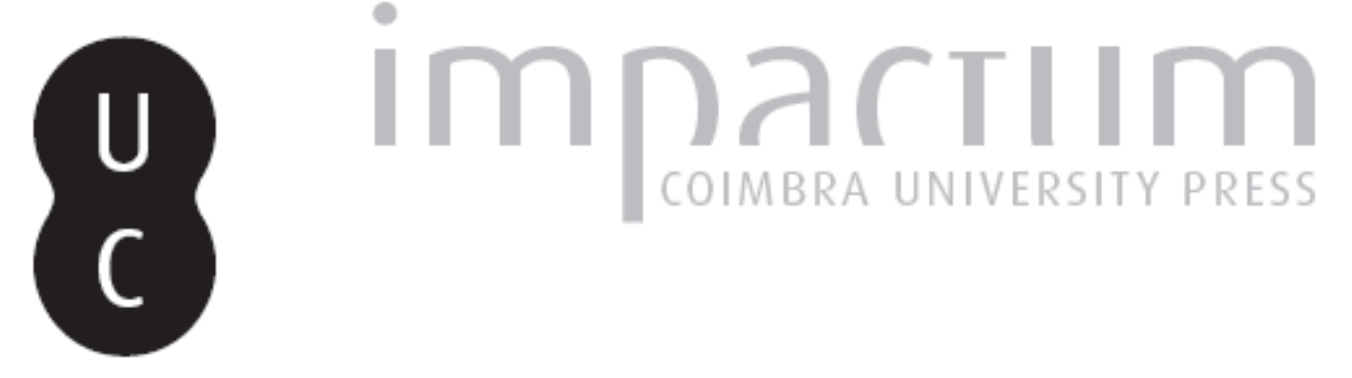

\title{
L'exègèsis chez Plutarque: textes poétiques, textes philosophiques et vérité
}

Autor(es): Bréchet, Christophe

Publicado por: International Plutarch Society

URL persistente:

URI:http://hdl.handle.net/10316.2/37655

DOI:

DOI:http://dx.doi.org/10.14195/0258-655X_8_4

Accessed : $\quad$ 26-Apr-2023 11:56:56

A navegação consulta e descarregamento dos títulos inseridos nas Bibliotecas Digitais UC Digitalis, UC Pombalina e UC Impactum, pressupõem a aceitação plena e sem reservas dos Termos e Condições de Uso destas Bibliotecas Digitais, disponíveis em https://digitalis.uc.pt/pt-pt/termos.

Conforme exposto nos referidos Termos e Condições de Uso, o descarregamento de títulos de acesso restrito requer uma licença válida de autorização devendo o utilizador aceder ao(s) documento(s) a partir de um endereço de IP da instituição detentora da supramencionada licença.

Ao utilizador é apenas permitido o descarregamento para uso pessoal, pelo que o emprego do(s) título(s) descarregado(s) para outro fim, designadamente comercial, carece de autorização do respetivo autor ou editor da obra.

Na medida em que todas as obras da UC Digitalis se encontram protegidas pelo Código do Direito de Autor e Direitos Conexos e demais legislação aplicável, toda a cópia, parcial ou total, deste documento, nos casos em que é legalmente admitida, deverá conter ou fazer-se acompanhar por este aviso.

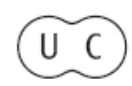




\title{
L'exègèsis chez Plutarque : \\ textes poétiques, textes philosophiques et vérité \\ par \\ Christophe Bréchet \\ Université de Paris Ouest Nanterre La Défense / \\ UMR7041 ArScAn (THEMAM) \\ christophe.brechet@u-paris10.fr
}

\begin{abstract}
Although both poetic texts and the writings of Plato require "explanation" (exegesis), the exegesis of Homer will necessarily produce different results from that of Plato, given the very different relationship of each type of text to the truth. Poetic texts are the domain of the grammatikos, but his "explanation" is not sufficient to ensure that the reader will benefit or that poetry will serve as a propaedeutic to philosophy. To arrive at the truth, it is not enough simply to explain the meaning of Homer's words or to clarify his thought but, rather, his poetry must be constantly compared to the truth, which lies outside it. Far from being an explanation of the poets, the De audiendis poetis therefore teaches the reader above all to adopt a critical approach to poetry. By contrast, the truth is to be found in the writings of Plato, which allow the reader to understand the world as a whole and provide a basis for understanding the essential truths. In the case of Platonic texts, Plutarch offers his own exegesis. He writes for his sons and his friends, providing them with an ethics of exegesis, for exegesis is a means not just of approaching the content of a work and its doctrines but also of putting the exegete himself to the test. Its ultimate goal is to make the exegete, as far as possible, at one with himself, with Plato and with the truth.
\end{abstract}

Key-Words: Exegesis, Poetry, Philosophy, Truth, Meaning.

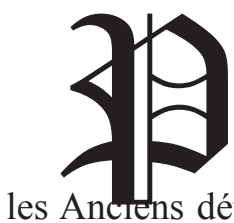

endant longtemps, les spécialistes de l'exégèse se sont tellement intéressés à la façon dont de leurs mythes qu'ils en ont oublié que l'exégèse était, avant tout, l'explication d'un texte $^{1}$ : pour eux, l'exégèse la plus riche - l'exégèse par excellence avait le mythe pour objet et l'allégorie comme méthode interprétative. Cette double réduction est allée de pair avec

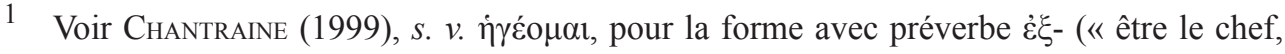

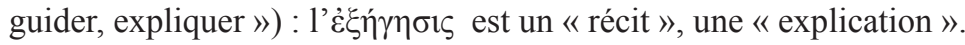


l'idée qu'on pouvait regrouper sous un concept unique - le mythe - la diversité des mythes antiques, et que ce qui était valable pour le mythe en général l'était a fortiori pour un mythe particulier. Une telle focalisation sur le mythe conçu comme unitaire a eu pour résultat d'occulter la singularité des pratiques exégétiques, d'un auteur à l'autre, et, partant, des regards que les auteurs antiques pouvaient porter sur un même texte.

L'analyse qu'on a pu faire de l'exégèse d'Homère chez Plutarque me semble un exemple remarquable de cette tendance. Les deux ouvrages des années 1950 qui ont fait date, Les mythes d'Homère et la pensée grecque de Félix Buffière $^{2}$ et Mythe et Allégorie. Les origines grecques et les contestations judéo-chrétiennes de Jean Pépin ${ }^{3}$, ont en commun de présenter l'Iliade etl'Odyssée comme des mythes dont Plutarque ferait une exégèse sinon allégorique, du moins morale ${ }^{4}$. Or, l'auteur du De audiendis poetis, loin de vouloir mettre au jour le sens moral immanent des vers d'Homère - tant il est clair, pour lui, que beaucoup de vers sont en soi immoraux -, montre simplement que la poésie homérique a une utilité dans la formation morale et intellectuelle du jeune Grec, pour peu qu'on lui apprenne à adopter une attitude critique vis-à-vis d'Homère : l'important, ce n'est pas le sens d'un vers, mais la façon dont on peut l'utiliser" ${ }^{5}$. Ce n'est donc pas un traité d'exégèse qu'il écrit, mais un manuel d'utilisation des poètes.

Afin de ne pas rester dans notre vision de l'exégèse - que nous précisons volontiers par un adjectif : exégèse littérale, historique, allégorique, etc.--, je me propose ici de repartir des occurrences des mots de la famille d'exègèsis chez Plutarque pour déterminer ce qui, dans son esprit, nécessite une « explication » - tel est, fondamentalement, le sens

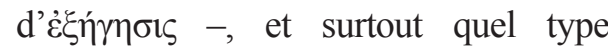
d'explication. L'exègèsis, rappelonsle, occupait une grande place dans le cursus scolaire. Comme le rappelle H.I. Marrou, « le travail du grammairien sur un auteur se divise en quatre opérations : la critique du texte, la lecture, l'explication,

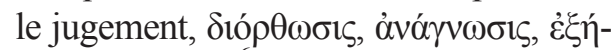

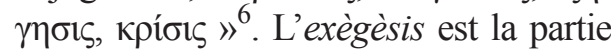
prépondérante du travail, notamment sur Homère. Elle est requise quand le sens n'est pas transparent, ne serait-ce que parce que le vocabulaire employé est

3 PÉPIN (1958).

4 Buffière (1956), p. 253sqq. et PÉPIN (1958), p. 181 sqq.

5 Voir BRÉCHET (2007).

6 Marrou (1965), p. 250. Voir aussi Denys le Thrace, Ars grammatica 1, 1, 4-8, et Sextus Empiricus, Adversus Mathematicos, 1, 251 sqq. 
éloigné de l'usage courant ( $\sigma u v \eta ́ \theta \varepsilon ı)$ : ce n'est alors que par le biais de l'exègèsis que l'absence de clarté (àoó$\varphi \varepsilon i \alpha)$ qui en résulte peut laisser place à la clarté $(\sigma \alpha \varphi \eta ́ v \varepsilon i \alpha)^{7}$. Or le terme exègèsis, inséparable de l'explication d'Homère $^{8}$, n'apparaît qu'une fois dans le De audiendis poetis. En revanche, son emploi est mieux établi pour les textes de Platon, à commencer par le Timée.

Pourquoi l'auteur des Euvres Morales voit-il dans les dialogues de Platon plus que les poèmes d'Homère une matière à exègèsis de sa part ? Comme je voudrais le montrer, ce qui est en jeu, c'est, en même temps que le statut respectif des textes poétiques et des textes philosophiques, la façon dont Plutarque conçoit son rôle de guide ou d'exègètès, pour son entourage. Il consacre certes un traité à l'audition des poètes, mais les textes poétiques ne sont qu'une propédeutique à l'étude des textes philosophiques, et ce sont ces derniers qui réclament la véritable exègèsis.

\section{Les mots de la famille d'exègèsis chez Plutarque}

Les mots de la famille d'exègèsis sont présents dans près de 35 textes, chez Plutarque ${ }^{9}$. Très souvent, il est question d'interpréter des choses sacrées. On rencontre ainsi, dans les Vies parallèles, toute une série d'exégètes, tels des pontifes ou des mages ${ }^{10}$, qui viennent rappeler que l'exègètès a d'abord été l'interprète des rêves, des signes, des oracles, bref, de tout ce qui était obscur et réclamait la médiation d'un spécialiste. Dans la Vie de Nicias $(23,9)$, Plutarque évoque par exemple l'ouvrage qu'Autoclide avait consacré aux traditions religieuses : les Exégétiques.

7 Un des grands principes des Alexandrins, rappelons-le, consiste à « éclairer Homère par

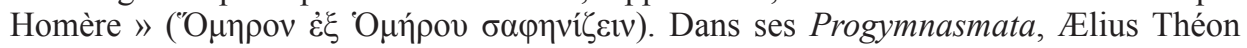
explique qu' " en ce qui concerne les mots, celui qui recherche la clarté ( $\tau \tilde{\omega}$ б $\sigma \alpha \varphi \eta v i ́ \zeta o v \tau \imath)$ doit éviter l'emploi des mots poétiques, forgés, métaphoriques, archaïques, étrangers et homonymes » (81, 8-10). Pour les mots poétiques, les exemples sont des mots homériques : «Sont poétiques tous ceux qui nécessitent une explication ( $\dot{\xi} \xi \gamma \eta \dot{\sigma \varepsilon} \omega \varsigma)$ : krêgyon (vérité), alegizein (ne pas s'inquiéter de), marnasthai (combattre) et autres semblables ». Voir aussi, sur ce lien entre exègèsis et saphèneia, Denys d'Halicarnasse, Thucydide 54, 24-27.

Voir notamment l'importance des mots de la famille d'exègèsis dans les scholies à Homère

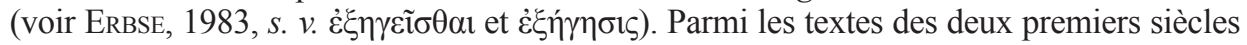
où il est question d' " expliquer Homère " ("O Pruse, Orat. 2, 79, 6 ; Aelius Théon, Progymn., 129, 26 ; Alexandre d'Aphrodise, Comment.

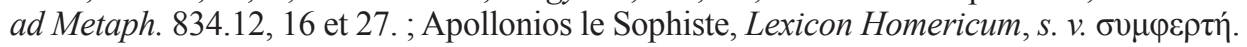

9 Les occurrences sont réparties à part presque égale entre les Euvres morales et les Vies parallèles. Je laisse de côté les trois fragments $(41.2,65.3$ et 90.3) où il est question de Plutarque exégète, car ils nous renseignent uniquement sur la réception de cet auteur.

10

Ceux-ci procèdent parfois comme les exégètes de textes « littéraires » (voir De Iside, 362C).

Ploutarchos, n.s., 8 (2010/2011) 35-50

ISSN 0258-655X 
Les faits religieux ne sont pas les seuls à nécessiter une exégèse. Dans les Questions grecques (4, 292B), Plutarque fait référence à une stèle au sens obscur, dont Aristote avait proposé une explication. Dans le De Iside (12, 355C), c'est la mythologie égyptienne qui nécessite une exégèse. Mais il ne s'agit plus, alors, de clarifier ce qui est obscur. Le sens littéral est rejeté :

Par conséquent, lorsque tu auras entendu ce que la mythologie égyptienne raconte sur les dieux : errances, démembrements et autres malheurs du même genre, tu devras te souvenir de ce qui vient d'être dit et te garder de croire que cela corresponde tel quel à un événement ou à un fait réel. Si les Egyptiens appellent Hermès « le chien », ce n'est pas au sens propre du mot (oủ... кupícs), mais parce que les qualités de cet animal, bon gardien, vigilant, philosophe, selon le mot de Platon (Rép. 375e), en ce qu'il distingue l'ami de l'ennemi en connaissant l'un et en traitant l'autre comme un inconnu, leur inspirent un rappro- chement avec le plus intelligent des dieux. Ils ne croient pas davantage que le soleil sorte d'un lotus sous la forme d'un nouveau-né : ils représentent ainsi le lever de l'astre pour symboliser

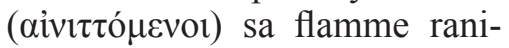
mée émergeant des eaux [...]. Eh bien ! c'est ainsi qu'il faut entendre ce qu'on dit des dieux. Reçois maintenant le mythe de ceux qui en donnent une interprétation conforme à la fois à la piété et à la philosophie ( $\tau \tilde{\omega} v$

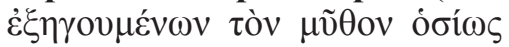

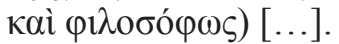

Ailleurs, ce sont des textes grecs qui sont explicitement présentés comme des objets d'exégèse ${ }^{11}$. Deux catégories se dessinent: les textes poétiques et les textes platoniciens. Dans la première catégorie, sont concernés les allusions dans la comédie ancienne ${ }^{12}$, un vers d'Alcman ${ }^{13}$ et deux expressions homériques ${ }^{14}$. Dans la seconde catégorie, on trouve les théories mathématiques de Platon ${ }^{15}$ et surtout le Timée ${ }^{16}$. Chez le Platonicien qu'est Plutarque, le premier auteur à réclamer une exégèse est, sans surprise, Platon.

11 Je laisse de côté les passages qui ne mettent pas en jeu l'explication des textes, et où le verbe exègeisthai signifie simplement « expliquer en détail ».

13 De facie, 940A.

14 De audiendis poetis, 31E; Amatorius, 757B.

15 De defectu oraculorum, 427A

16 Platonicae quaestiones, 1006F ; De animae procreatione in Timaeo, 1012B; 1012C; 1014A. Je laisse de côté le De fato (568F), jugé inauthentique : il y est question du mythe crypté de la République. 
Il est intéressant de regarder qui mène l'exégèse, selon le texte. Pour les textes poétiques, c'est avant tout le professeur de lettres ou grammatikos qui officie. Dans les Propos de table (VII, 8, 711F-712A), Plutarque fait remarquer que les allusions à la démocratie athénienne contemporaine sont si fréquentes dans la comédie ancienne qu'elle n'est plus directement compréhensible, pour les époques ultérieures :

En outre, de même que dans les dîners des grands, chacun à table a auprès de lui un échanson, de même il faudra à chacun son professeur de littérature ( ( $\dot{\xi} \xi \eta \gamma \varepsilon \tilde{\sigma} \sigma \alpha \alpha)$ chaque détail : qui est Laispodias chez Eupolis, Cinésias chez Platon, Lampon chez Cratinos, et ainsi pour chacun des personnages brocardés, si bien que notre banquet deviendra une salle de classe ou que les traits se perdront sans pointe ni signification.

L'exégèse, ici, relève de l'érudition. Dans le De facie (940A), c'est le grammairien Théon qui propose une exégèse physique d'un vers d'Alcman. Dans les deux autres passages, les exégètes sont des stoïciens, et dans les deux cas, il est question d'expressions homériques. Plutarque rapproche alors ces champions de l'étymologie sophistiquée des grammatikoi. Dans le De audiendis poetis (31D-F), il écrit qu'il n'est pas convaincu par les plaisanteries des stoïciens :
S'il ne faut pas écouter négligemment les mots, il faut également refuser les plaisanteries de Cléanthe, car parfois, il se moque en feignant d'interpréter ( $\dot{\varepsilon} \xi \eta \gamma \varepsilon \tilde{\sigma} \sigma \theta \alpha)$ l'expression « Zeus père qui exerces ton pouvoir sur l'Ida » comme « qui exerces ton pouvoir par l'idée », et quand il veut qu'on lise l'expression «Zeus, seigneur de Dodone » en un seul mot, la vapeur qu'il s'exhale de la terre étant selon lui " anadodonéenne » puisqu'elle fait une " anadosis » (" montée »). Chrysippe aussi, dans bien des cas, donne dans les subtilités ; il ne plaisante pas, mais trouve beaucoup d'explications peu convaincantes, forçant le sens pour que « le fils de Cronos à la parole qui porte loin » signifie que Zeus est habile à discuter et surmonte tous les obstacles par la force de sa parole. Il vaut mieux laisser ces finesses aux grammai-

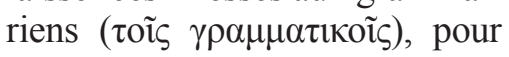
insister plutôt sur les vers qui contiennent des idées profitables et dignes de créance.

L'excès de subtilité est pardonnable quand on plaisante, mais répréhensible quand on se prend au sérieux. Chrysippe, ici, se voit adresser un triple reproche : d'abord, il s'attache à des détails ( $\gamma \lambda i ́ \sigma \chi \rho o \varsigma)$; ensuite, il fait preuve d'une habileté excessive dans ses explications

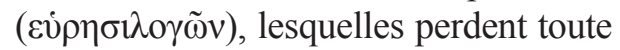

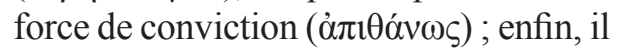
fait violence au texte ( $\pi \alpha \rho \alpha \beta 1 \alpha \zeta o ́ \mu \varepsilon v o \varsigma)$. 
Plutarque ne veut pas que les jeunes gens pratiquent ce type d'exégèse : la bonne attitude, selon lui, consiste à réfléchir sur un passage problématique et à comprendre pourquoi il est problématique, plutôt que de chercher à le rendre moral par des moyens intellectuellement contestables. C'est là un point très important : quand le sens des vers est immoral, l'exègèsis doit être prolongée par une activité critique où il n'est plus question d'éclairer le sens du vers dans le contexte, ni de feindre que le vers signifie autre chose, mais de dire pourquoi, dans son rapport à la vérité, le vers est incorrect et, éventuellement, de le corriger ${ }^{17}$.

On aperçoit ici un aspect important de l'exégèse, qui n'est pas seulement explicitation d'un sens, mais qui est porteuse d'une certaine attitude d'esprit : l'exégèse ne doit pas être un fourvoiement de l'intelligence. C'est encore plus net dans l'Erotikos (757B), où Chrysippe se fait l'exégète du nom d'Arès :

Chrysippe, en expliquant ( $\dot{\varepsilon} \xi-$

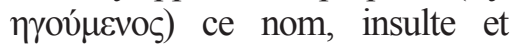
calomnie la divinité : il prétend qu'Arès signifie « Le Tueur ». Chrysippe ouvre ainsi la voie à ceux qui pensent qu'Arès est le nom de cette tendance au com- bat, à la dispute et au courage qui existe dans chacune de nos âmes. D'autres, à leur tour, prétendront qu'Aphrodite, c'est le désir ; Hermès, la parole ; les Muses, les arts ; Athéna, la sagesse. Vois-tu bien l'abîme d'athéisme où nous tomberons, si nous réduisons chacun des dieux à personnifier nos passions, nos facultés et nos vertus?

Si l'exègèsis de Chrysippe est rejetée, c'est parce qu'elle entraîne la disparition des dieux, identifiés aux passions humaines.

Les exégèses pratiquées sur les textes poétiques, par les Stoïciens ou les grammairiens, illustrent donc tantôt ce qu'il ne faut pas faire, tantôt ce qui est insuffisant ${ }^{18}$. On retiendra ici la réticence de Plutarque à employer les mots de la famille d'exégèsis quand il cherche, lui, à tirer profit des poètes, en particulier d'Homère. La raison en est simple : à partir du moment où on admet que les poètes n'ont pas vocation à dire vrai, et qu'on veut toutefois les utiliser dans la formation morale des jeunes gens, il faut recourir à d'autres procédés qu'à la stricte explication des vers ${ }^{19}$.

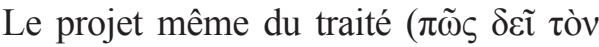

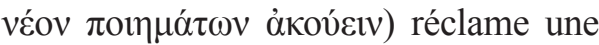
panoplie de méthodes qui dépasse, de très loin, le champ de l'exégèse.

18 Voir De audiendis poetis, 22C.

19 La section du De audiendis poetis consacrée aux gloses de mots difficiles (22C-25B) est ainsi assez réduite. 
Venons-en aux textes platoniciens. Si on excepte le De defectu oraculorum (427A), où il est question de l'exégèse que Théodore de Soles a faite des théories mathématiques de Platon, c'est Plutarque lui-même qui parle de son interprétation de Platon en termes d'exègèsis. C'est en ce sens que je disais qu'il est intéressant de regarder qui est le praticien de l'exégèse, dans chaque texte. Le passage le plus connu est sans doute l'ouverture du De tranquillitate animi (464E) :

C'est trop tard que j'ai reçu ta lettre, par laquelle tu m'invitais à t'écrire sur la tranquillité de l'âme et sur certains passages du Timée qui réclament une exégèse plus approfondie

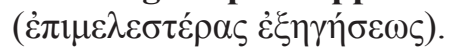

On ne sait pas exactement à quel traité Plutarque fait allusion ${ }^{20}$, même si on a voulu parfois y reconnaître le long commentaire sur La génération de l'âme dans le Timée de Platon, ou les Questions platoniciennes. On peut d'ailleurs s'autoriser du fait que dans ces deux dernières œuvres - sur lesquelles je vais revenir -, Plutarque emploie justement les mots de la famille d'exègèsis (respectivement trois et une fois).

Il suffit, pour l'instant, de noter qu'il n'est guère que deux objets dont Plu- tarque reconnaisse explicitement faire l'exégèse : la mythologie égyptienne et les textes platoniciens. Ces deux exégèses, disons-le tout de suite, diffèrent non seulement par l'importance qu'elles ont eue dans l'activité de Plutarque - le début du commentaire sur le Timée montre que l'exégèse platonicienne a été, pour lui, une activité beaucoup plus fréquente $^{21}-$, mais aussi par leur nature - la première est nettement mystique, la seconde, plus littérale. Si d'autres auteurs, et notamment des néoplatoniciens, ont rapproché le Poète et le Philosophe et fait d'Homère un théologien, tel n'est pas le cas de Plutarque ${ }^{22}$.

Compte tenu du petit nombre d'occurrences des mots de la famille d'exègèsis, nos conclusions peuvent sembler contestables. On arrive cependant aux mêmes conclusions à partir d'autres études lexicales, en partant de termes qui engagent également le statut des textes. Je songe ici à deux familles : celle d'ainigma et celle de théologia. Leur étude montre sans ambiguité que Plutarque considère Platon comme un théologos, de même qu'Hésiode, mais pas Homère, qui est avant tout un poète. Pareillement, Plutarque parle d'énigmes à propos de philosophes et d'Hésiode, mais il ne parle guère d'ainigmata homériques ${ }^{23}$.
20 Voir Pettine (1984), p. 91.
21 Voir aussi, pour la liste des ouvrages consacrés à l'exégèse de Platon, SiRINELLI \& IRIGOIN (1987), «Introduction générale », p. CXXV.
22 Voir BrÉCHET (2003), p. 420-454, et BRÉCHET (2005).
23 Ibid., p. 448-449.

Ploutarchos, n.s., 8 (2010/2011) 35-50

ISSN 0258-655X 
Dès lors, chez Plutarque, les mythes d'Homère ne peuvent être interprétés comme des textes cryptés et qui requerraient, de ce fait, un dépassement du sens littéral. Comme le dit Plutarque, au chapitre 20 du De Iside et Osiride (358E-359A), il y a deux types de mythes : ceux qu'inventent les poètes, comme une araignée tisse sa toile - les mythes d'Homère relèvent de cette première catégorie -, et ceux qui ont un sens profond et nous renvoient à autre chose - c'est le cas de la mythologie égyptienne. Le texte mérite d'être cité :

Voilà à peu près l'essentiel du mythe, à l'exclusion des épisodes les plus choquants, comme le démembrement d'Horus et la décollation d'Isis. Si, à propos de la nature bienheureuse et incorruptible - en cela se résume la conception du divin -, les Égyptiens croient et racontent tout cela comme autant d'actions et de faits authentiques, alors inutile de te dire, avec Eschyle, qu' « il faut tout recracher et se rincer la bouche », car tu es la première à t'indigner contre ceux qui ont sur les dieux des opinions aussi anormales et barbares. Mais que, d'autre part, tout cela ne ressemble aucunement aux fables chétives, aux fictions inconsistantes que poètes et prosateurs tissent et tendent comme les araignées font leur toile, en en tirant de leur propre fonds les données arbitraires, et prend en compte des détails troublants et des passions vécues, tu seras la première à l'admettre. Et de même que, selon les savants, l'arc-en-ciel est une image du soleil qui doit d'être diaprée à ce que le rayon visuel est réfracté et revient se fixer sur le nuage, de même le mythe est, dans le cas qui nous occupe, le reflet d'un logos qui réfracte notre pensée vers d'autres choses [...].

L'activité créatrice du poète, comme Plutarque l'explique dans le De audiendis poetis, est tout entière tendue vers le plaisir de l'auditeur ou du lecteur, et non vers la vérité : il n'y a donc pas lieu d'y rechercher ce qui ne s'y trouve pas. Pour autant, cela n'interdit pas qu'on en retire quelque profit moral, comme le montre Plutarque à partir des mythes homériques les plus critiqués.

\section{Un exemple d'interprétation de mythes homériques}

Plutarque, dans la lignée de Platon (Rép. III 378d), condamne l'interprétation allégorique des poètes, notamment au chapitre 4 de son $D e$ audiendis poetis (19E-20B) :

Mais les poètes proposent d'autres enseignements à tirer des faits eux-mêmes, comme Euripide, dit-on, déclara à ceux qui blâmaient son Ixion pour son impiété et sa scélératesse : « Mais je ne l'ai fait sortir de scène qu'après l'avoir cloué à la roue ». Chez Homère, ce 
genre d'enseignement est implicite : il postule un examen approfondi utile à faire pour les mythes les plus critiqués, dont certains commentateurs, avec ce qu'on appelait autrefois " sens sous-jacents " et qu'on appelle maintenant des " interprétations allégoriques "

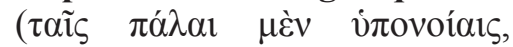

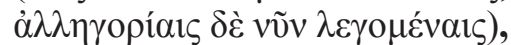
torturent et faussent la signifi-

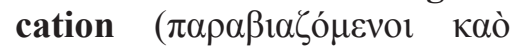
$\delta 1 \alpha \sigma \tau \rho \varepsilon \dot{\varphi}(\sigma o v \tau \varepsilon \varsigma)$, en prétendant que le soleil dénonce les amours adultères d'Aphrodite avec Arès (Od., 8, 270 sqq.) en ce sens que la conjonction de l'astre d'Aphrodite avec celui d'Arès produit des naissances adultérines et que, lorsque le Soleil recommence sa course et les surprend, elles ne restent pas secrètes. Pour la parure que revêt Héra à l'intention de Zeus, et le pouvoir magique attaché à se ceinture (Od., 14, 170 sqq.), ils veulent que ce soit une purification de l'air s'approchant de la matière ignée, comme si le Poète ne donnait pas lui même les solutions. En effet, dans le passage concernant Aphrodite, il veut apprendre aux lecteurs attentifs que la musique immorale, les vilaines chansons, les entretiens qui portent sur des sujets indécents, engendrent des mœurs relâchées, des existences effé- minées, des hommes qui aiment la vie sensuelle, la mollesse, les rapports amoureux avec les femmes, " les vêtements souvent changés, les bains chauds et les plaisirs du lit» (Od., 8, 249). Aussi a-t-il représenté Ulysse ordonnant à l'aède : " Allons, s'il te plaît, change de sujet et chante la construction du cheval » (Od., 8, 492), faisant bien comprendre que les artistes et les poètes doivent trouver leurs sujets auprès de ceux qui sont intelligents et sensés. Et dans le passage concernant Héra, il a montré à la perfection que, si l'on doit à des drogues et à la magie, si l'on obtient par supercherie, les relations intimes avec les hommes et leur faveur, on inspire un sentiment qui non seulement dure peu, trouve vite sa satiété et manque d'assise, mais même se change en haine et en colère quand les attraits du plaisir sont fanés. Voici en effet les menaces que Zeus adresse à Héra : « Ainsi tu vas voir ce qu'ils t'auront rapporté, cet amour et ce lit où tu es venue loin des dieux t'unir à moi et me duper » (Il., 15, 32-33).

Plutarque refuse ici l'allégorie physique des Stoïciens ${ }^{24}$, parce qu'elle change la signification de ce texte : le texte poétique, dans le cas d'Homère, ne signifie pas autre chose que ce qu'il exprime, le poète n'étant pas investi 
d'un savoir sur le kosmos. Quelle lecture, alors, Plutarque propose-t-il ?

L'épisode des amours d'Arès et d'Aphrodite est, en soi, est immoral : Homère y a représenté la divinité de façon indigne. Plutarque déplace donc l'attention du lecteur du contenu du mythe à sa réception à l'intérieur même du poème, en montrant que tout le monde n'écoute pas l'aède Démodocos de la même façon : les Phéaciens, esclaves du plaisir, prennent plaisir à l'entendre chanter cet épisode, tandis que le très vertueux Ulysse lui demande de prendre un sujet plus décent. Certains accuseront Plutarque sinon de manipuler le texte, du moins de ne pas en respecter la linéarité ${ }^{25}$. Mais son interprétation prend en compte la façon dont Homère met en scène certains épisodes, dans l'Odyssée. En soi, le mythe est immoral, mais sa réception, dans l'économie $\mathrm{du}$ poème, est acceptable, pour peu qu'on se concentre sur le personnage d'Ulysse. On est, ici, aux antipodes des exégèses qui considèrent que ce mythe est crypté : qu'on songe aux multiples interprétations allégoriques qu'on trou- ve dans le De Homero ou dans les Allégories d'Homère ${ }^{26}$.

Pour Héra séduisant Zeus, là encore, le mythe ne signifie pas autre chose que ce qu'il dit, pour peu qu'on le lise jusqu'au bout : quand une femme obtient les faveurs d'un homme par la tromperie, elle ne doit rien attendre de bon de la relation instaurée. Pour Plutarque, il faut exploiter les vers $I l ., 15$, $32-33^{27}$, qui montrent qu'Héra est bel et bien punie de sa traîtrise. Là encore, on est très loin des Allégories d'Homère (39), où le contenu philosophique des épopées homériques est sans cesse souligné : si Plutarque dégage un sens moral, il ne transpose jamais, lui, au plan physique ou métaphysique.

Pour conclure sur ces deux passages, Plutarque ne substitue pas une allégorie morale à une allégorie physique, comme on a pu le prétendre ${ }^{28}$ : les interprétations qu'il propose sont étrangères à l'allégorie. L'idée est qu'il faut repérer les « enseignements à tirer des faits eux-mêmes » (19D), être attentif à « ce genre d'enseignement implicite » (19E). Pour le dire plus clairement,

Plutarque se fonde ici sur un vers qui apparaît 130 vers après l'épisode des amours d'Arès et d'Aphrodite et qu'il extrait de son contexte. Juste après l'épisode, rappelons-le, Homère nous dit qu'Ulysse a pris autant de plaisir à écouter l'aède que les Phéaciens $(O d ., 8,367$ 9), et ce n'est que bien plus tard qu'il interpelle Démodocos et lui demande de poursuivre ses chants admirables par l'histoire du cheval de bois.

Héraclite, Allégories d'Homère, 69 ; [Ps.-Plutarque] De Homero, 102.

27 Plutarque ne cite bien entendu que les derniers vers de la tirade de Zeus à Héra, au début du chant 15 , et se garde de faire la moindre allusion aux vers qui précèdent, tant ils sont indignes de Zeus.

28 Voir par exemple PÉPIN 1958, p. 181. 
l'immoralité d'un mythe est neutralisée dès lors qu'on peut trouver, dans le poème, une condamnation potentielle de ce mythe. La méthode peut sembler artificielle à un lecteur moderne, mais pour un Ancien, cette interprétation, pour habile qu'elle soit, ne fait qu'exploiter les potentialités du texte.

Ce détour par l'interprétation de deux grands mythes d'Homère, que Plutarque n'aborde pas en termes d'exègèsis, éclaire, comme je voudrais le montrer, l'exégèse qu'il fait de Platon. Les parallèles sont nombreux avec le De audiendis poetis, ce qui tient au statut de texte de référence des deux corpus, dans le domaine poétique et dans le domaine philosophique, mais ils ne doivent pas masquer des différences essentielles.

\section{L'exègèsis de Platon telle que la conçoit Plutarque}

Le meilleur exemple de ce qui est explicitement présenté comme de l'exégèse par Plutarque est le De animae procreatione in Timaeo. Cet ouvrage est, à plusieurs titres, un manuel d'exègèsis, puisque Plutarque y adopte une méthode bien précise, exposant à ses fils les principes qui fondent son interprétation.

Je laisserai aux spécialistes de l'exégèse philosophique le soin d'expliquer comment Plutarque procède concrète- ment, dans son commentaire ${ }^{29}$, et ne m'intéresserai ici qu'aux passages où il commente son commentaire, en quelque sorte. Voici l'ouverture du traité (1012B) :

Puisque ce que j'ai dit et écrit çà et là pour expliquer l'opinion qu'avait Platon sur l'âme - du moins selon

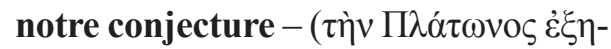

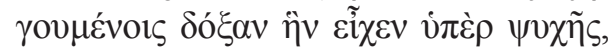
$\dot{\omega} \varsigma \dot{v} \pi \varepsilon v 0 o v \tilde{\mu} \varepsilon v \dot{\eta} \mu \varepsilon \tilde{\varsigma} \varsigma)$, doit, selon vous, être rassemblé en un seul ouvrage, et que cette explication doit recevoir un intitulé propre, parce qu'elle est tout sauf facile et qu'elle nécessite une persuasion du fait qu'elle s'oppose à la plupart de celles qu'ont apportées les successeurs de Platon, je commencerai par citer les mots mêmes du Timée.

Il cite alors Timée 35a1-b4, sur la composition de l'âme du monde, et mentionne toute une tradition exégétique (1012D) :

Commencer par passer en revue tous les désaccords que ce passage a occasionnés chez ceux qui ont cherché à

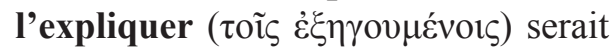
un travail infini, à présent, et comme vous en avez lu la plupart, il est également superflu.

Plutarque s'en tiendra donc à deux positions significatives : celle de Xénocrate et celle de Crantor de Soles. Après les avoir brièvement exposées, il souligne leur faiblesse (1013D) :

Voir notamment Donini (1992) et FERRARI (2001 et 2004).

Ploutarchos, n.s., 8 (2010/2011) 35-50

ISSN 0258-655X 
Tous les deux me semblent manquer l'opinion de Platon, s'il faut se servir du crédible comme d'une règle ( $\varepsilon i$ kavóvı

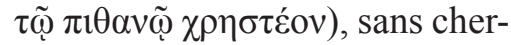
cher à parachever des théories personnelles, mais armés de la volonté de dire quelque chose qui s'accorde avec cet auteur

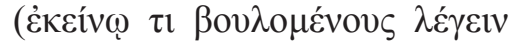

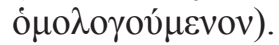

D'après ce passage, l'exègèsis réussie doit satisfaire à deux exigences. La première est la pithanotès, c'està-dire la crédibilité. On retrouve ici le reproche que Plutarque fait à Chrysippe dans le De audiendis poetis, en disant que ses pirouettes étymologiques n'entraînent pas l'adhésion $(\dot{\alpha} \pi \imath \theta \dot{\alpha} v \omega \varsigma)^{30}$. Or, c'est là un point crucial : à défaut de dire le vrai, il faut produire une explication qui soit crédible, autrement dit persuasive du fait de sa vraisemblance. A plusieurs reprises, notamment dans les Propos de table, il est question de ce critère à propos de l'interprétation de Platon $^{31}$. La deuxième exigence est l'homologia entre l'interprétation et le texte interprété, ou plus exactement entre l'interprète et l'auteur interprété : il doit y avoir « accord », « conformité ».
S'il s'agit d'une conformité logique, on peut aussi, je crois, songer à l'exigence d'homologia telle qu'elle apparaît dans les dialogues de Platon, en particulier dans le Gorgias. L'entretien dialectique, en effet, a pour but de mettre l'interlocuteur de Socrate en accord avec lui-même, mais aussi de parvenir à un accord entre les deux interlocuteurs, qui leur permette, chaque fois qu'un point a été admis de part et d'autre, de progresser méthodiquement dans la discussion et dans la recherche de la vérité (486e-488a). Dans le Commentaire sur le Timée, le terme suggère, je crois, que l'interprète n'est jamais seul, face au texte : il doit faire comme si l'auteur était là, en s'assurant, pour ainsi dire, que l'interprétation qu'il propose puisse recevoir son assentiment. En son absence, c'est en procédant à des rapprochements avec d'autres passages de son œuvre qu'il établira cet accord.

On peut, à partir des reproches que Plutarque adresse aux mauvais exégètes de Platon au chapitre 4, compléter la description de l'exègèsis réussie. La saphèneia est le but de l'exercice ${ }^{32}$ : le premier tort des mauvais exégètes est précisément de ne pas éclaircir un

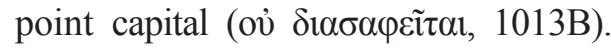

$3031 \mathrm{E}$, cité supra.

31 Voir notamment Quaestiones convivales VII, 1, 700B ; QC VIII, 8, 728E ; QC VIII, 2, 719E ; De defectu oraculorum 430B ; De animae procreatione in Timaeo, 1014A.

32 Voir De animae procreatione in Timaeo, 1012D et 1022A. 
L'exègèsis de Platon consiste ensuite à expliquer Platon par Platon ${ }^{33}$. Si certains n'ont pas compris la différence entre le Même et l'Autre (1013D), Plutarque, lui, invoque le Sophiste 254d4-259b7. Et tout au long de son commentaire, il multiplie les citations de Platon. C'est que Platon permet de sortir de contradictions apparentes : tout comme Homère dans le De audiendis poetis, il apporte lui-même la solution (lusis) aux problèmes que croient déceler ses lecteurs (1016A). Chez Homère comme chez Platon, il faut donc savoir dans quel sens les mots sont employés, en procédant à des rapprochements d'occurrences. Enfin, l'exègèsis s'accommode mal des violences faites au texte. On retrouve le verbe

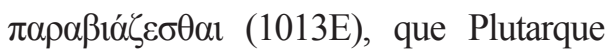
emploie pour les violences que Chrysippe fait au texte homérique ${ }^{34}$. Le bon exégète est donc celui qui éclaire le sens d'un texte sans faire violence à ce texte.

Dans le cas présent, éclairer le sens $\mathrm{du}$ texte sans lui faire violence, c'est accepter la génésis de du kosmos. En effet, un des problèmes qui se posait aux commentateurs de Platon était la discordance ( $\dot{\alpha} \sigma u \mu \varphi \omega v i ́ \alpha)$ entre le Phèdre (245c-246a), où l'âme est inengendrée et éternelle, et le Timée (34b-35a), où elle est engendrée et fabriquée $^{35}$. Au tout début de son Commentaire sur le Timée, Plutarque cite le texte du Timée (35a1-b4) puis prend le texte à la lettre : si Platon écrit, dans le Timée, que l'âme du monde a été engendrée, alors elle a été engendrée et le monde a bien eu une naissance ${ }^{36}$. L'interprétation de Plutarque est caractéristique d'un rationalisme philologique, qui repose sur une certaine conception du texte platonicien : ce dernier est suffisant et dit le vrai (fût-ce parfois obscurément, ce qui nécessite alors une exégèse).

Après cette doxographie, Plutarque en vient, au chapitre 5 , à son opinion personnelle, redonnant les principes qui fondent son exégèse :

D'abord, donc, je vais exposer la pensée qui est la mienne en ces matières, m'en remettant au crédible et en écartant, autant qu'il est possible, l'étrangeté et le paradoxe des propos ; ensuite, je confronterai mon explication et ma démonstration aux mots, en même temps que je les accorderai les uns aux autres. C'est ainsi qu'il en va,

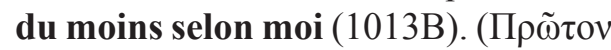

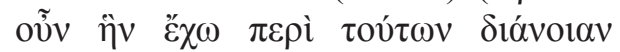

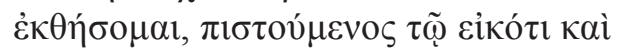

33 Les Alexandrins avaient fixé cette règle d'interprétation non seulement pour Homère, mais aussi pour les autres auteurs.

34 De audiendis poetis (31D-F), cité supra.

35 FERRARI (2001), p. 262-266.

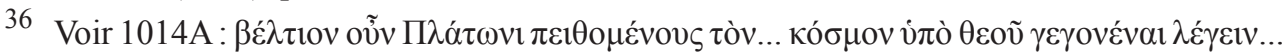

Ploutarchos, n.s., 8 (2010/2011) 35-50

ISSN 0258-655X 


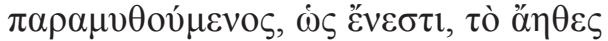

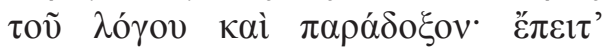

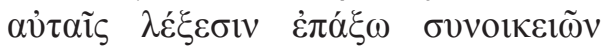

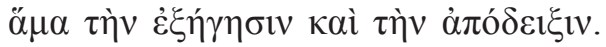

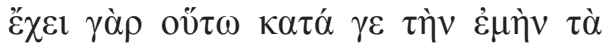
$\pi \rho \alpha ́ \gamma \mu \alpha \tau \alpha \delta o ́ \xi \alpha v$.)

On retrouve la vraisemblance, la conformité avec le texte, mais aussi l'apport nécessaire de preuves (apodeixis).

Sans entrer dans le détail du commentaire, je ferai une dernière remarque. On retrouve, maintes fois au long du texte, la même restriction :

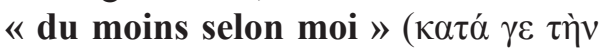

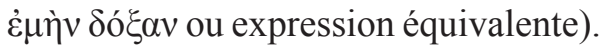
Il ne s'agit pas d'imposer son exégèse comme la meilleure, ni de faire preuve d'une assurance qui messied « sur des sujets où l'on voit les philosophes en proie au vertige », pour reprendre les mots du De audiendis poetis (17D). Plutarque montre les points faibles des autres exégètes et fonde son exposé sur les arguments les plus solides. On a là une différence essentielle avec l'interprétation des textes poétiques : sur des sujets aussi importants que ceux que traite Platon, l'exégète doit rester modeste, et ne pas oublier qu'atteindre le vrai n'est pas chose aisée, tant pour les philosophes que pour ceux qui tentent d'expliquer leurs textes. A ma connaissance, on ne trouve jamais une telle prudence pour les poètes. La raison en est simple : avec les textes poétiques, il n'est pas question de la vérité, mais seulement, serais-je tenté de dire, de la vérité du texte - ou de ce qu'on pourra présenter comme telle.

\section{Conclusion}

Pour conclure, par bien des aspects, Plutarque commente Homère et Platon de façon comparable. Il " explique Homère par Homère ", et "Platon par Platon ", sans recourir aux interprétations allégoriques, comme beaucoup d'auteurs le faisaient. L'explication menée est rigoureuse et doit beaucoup au commentaire philologique scolaire, qui repose sur l'éclaircissement du sens des mots et le rapprochement de passages. Dans les deux cas, on a l'idée qu'il y a un texte de référence et que celui-ci est suffisant. De ce point de vue, l'interprétation de Platon est plus proche de celle d'Homère que de celle de la mythologie égyptienne, où le sens littéral doit être dépassé.

Mais si on peut faire une exègèsis d'Homère et de Platon, il ne faut pas en attendre la même chose, parce que les deux textes n'entretiennent pas le même rapport avec la vérité. Au tout début du De audiendis poetis (15A), Plutarque écrit que les jeunes garçons ont besoin non pas d'une bonne exègèsis, quand ils écoutent les poètes, mais d'une bonne paidagôgia. Certes, avec exègeisthai et paidagôgein, il s'agit toujours de " guider », mais dans des perspectives différentes : si les pères (Plutarque et Marcus Sedatius, destinataire du traité), doivent se préoccuper de la façon dont 
leurs fils vont écouter les poètes, c'est que l'exégèse du grammatikos n'est pas suffisante pour que l'audition soit profitable et que la poésie soit une propédeutique à la philosophie. Il ne suffit pas d'expliquer le sens des mots, chez Homère, ou d'expliciter sa pensée, pour trouver la vérité : il faut sans cesse confronter ses vers à la vérité, qui leur est extérieure $^{37}$. Loin d'être un commentaire des poètes, le De audiendis poetis apprend avant tout à adopter une attitude critique vis-à-vis d'eux.

En revanche, la vérité est dans le texte platonicien, qui permet de saisir le monde dans son ensemble, et à partir duquel on peut comprendre les vérités essentielles. Si l'enseignement de la philosophie, à l'époque impériale, consiste de plus en plus à expliquer les textes des autorités, nous savons peu de chose de ce qui se passait dans les écoles. Plutarque nous offre ici un bel exemple de ce contact personnel avec le texte, en dehors des grandes écoles $^{38}$. Il écrit pour ses fils, ses amis, et leur livre même, je pense, une éthique de l'exègèsis. L'exégèse, en effet, permet non seulement de se confronter à un contenu doctrinal, mais aussi de s'éprouver en tant qu'exégète. Elle a pour but de mettre l'exégète, autant que faire se peut, en accord avec lui-même, avec Platon et avec la vérité, comme dans un entretien réel. Platon, d'ailleurs, est presque là, comme le montre la deuxième question du livre VIII des Propos de table (QC VIII, 2, 718C) : à l'occasion de l'anniversaire de Platon, on décide de « le faire participer en personne à la conversation en examinant en quel sens il a dit que Dieu ne cessait de faire de la géométrie ».

\section{BibLIOGRAPHIE}

BABUT, D.,

- Plutarque et le stoïcisme, Paris, Les Belles Lettres, 1969.

BRÉCHET, CHR.,

- Homère dans l'œuvre de Plutarque. La référence homérique dans les Euvres Morales, Thèse de doctorat, 2003.

- «La lecture plutarquéenne d'Homère : de la Seconde Sophistique à Théodore Métochite », dans La tradition des Moralia de Plutarque de l'Antiquité au début de la Renaissance, Pallas, 67 (2005) 175-201.

- «Vers une philosophie de la citation poétique : écrit, oral et mémoire chez Plutarque », dans Philosophia and Philologia : Plutarch on Oral and Written Language, Hermathena, 182 (2007) 101-134.

BufFIÈRE, F.,

- Les mythes d'Homère et la pensée grecque, Paris, Les Belles Lettres, 1956.

Chantraine, P.,

- Dictionnaire étymologique de la langue grecque, Paris, Klincksieck, 1999.

Donini, P.,

- «Plutarco e i metodi dell'esegesi filosofica ", dans I Moralia di Plutarco tra Filologia e Filosofia, I. Gallo \& R. Laurenti (ed.), Strumenti per la ricerca plutarchea 1, M. D’Auria, Napoli, 1992, pp. 79-96.

37 Tout au plus y a-t-il, sur tel ou tel point, coïncidence entre les écrits poétiques et la vérité (voir Erotikos, 20, 765D-E).

38 Voir SiRinelli (2000), p. 125sqq.

Ploutarchos, n.s., 8 (2010/2011) 35-50

ISSN 0258-655X 
ERbSe, H.,

- Scholia Graeca in Homeri Iliadem, vol. VI, Indices, Berlin, W. de Gruyter, 1983.

Ferrari, F.,

- « La letteratura filosofica di carattere esegetico in Plutarco », dans Orpheus, 22 (2001) 77-108.

- "Platone in Plutarco », dans La Biblioteca di Plutarco, Gallo, I. (ed.), Napoli, M. D’Auria, 2004, pp. 225-235.

Flacelière, R. \& Irigoin, J.,

- Plutarque, Euvres Morales, tome I. 1, Paris, Les Belles Lettres, 1987.
Marrou, H.-I.,

- Histoire de l'éducation dans l'Antiquité, Paris, Seuil, 1965.

PÉPIN, J.,

- Mythe et Allégorie. Les origines grecques et les contestations judéo-chrétiennes, Aubier, Editions Montaigne, 1958.

Pettine, E.,

- La tranquillità dell'animo di Plutarco, Salerno, Palladio, 1984.

SirinelLi, J.,

- Plutarque de Chéronée. Un philosophe dans le siècle, Paris, Fayard, 2000. 